\title{
The Lab-8 computer and quantitative methods
}

\author{
JOHN C. ARMINGTON \\ Northeastern University, Boston, Massachusetts 02115
}

\begin{abstract}
A method has been developed for teaching a graduate course that combines quantitative methods and introductory programming. It has produced an outcome that is of considerable utility in the laboratory. Several features that contribute to the success of this course are described.
\end{abstract}

New students come to graduate school eager to begin their research, but of ten lacking essential skills in mathematics and computer programming. This report touches on the highlights of a course that is designed to help them meet these deficiencies. It trains students in the conventional subject matter of quantitative methods as described by Daniels (1928), Guilford (1936), Lewis (1966), and Smith (1966) in an accelerated fashion using a computer to solve the problems. The value of the material presented in these rather old-fashioned texts is not easily overstressed, because it leads to an understanding of mathematical techniques that are widely applied in experimental psychology. Entering students frequently have a good background in elementary statistics, but it tends to be one of a "cookbook" sort. Specific statistical techniques may have been learned and some familiarity with prepackaged computer programs may have been gained, but few students have sufficient background to fully appreciate the specialized character and the limitations of the methods they are using. Deeper insights are needed.

The present course emphasizes curve fitting, plotting, and interpretation. While mastering this material, a student must review many aspects of mathematics such as algebra, matrices, solution of equations, logarithms, calculus, etc. Most of this is done with the support of a computer. Although the course cannot deal with all these topics thoroughly, it does build up confidence and prepare students to enter more advanced courses with a good working foundation.

There is another important feature. An incoming class of graduate students is seldom uniform. The work described here provides some common background. Actually, in fact, advantage is taken of the varied backgrounds of students by having them work in pairs or small groups, one member of each group being already experienced in computers, another in statistics, etc. The informal tutoring that takes place makes it possible to move forward at an accelerated pace.

The following discussion is separated into three parts. The first is a description of the system in current use. The second is an outline of the course, as well as of cer-

Research applications of the system described in this report were supported in part by PHS Grant EY 00589. tain of its programming techniques. The third is an overview of a practical program that was developed as a part of the course but which is now routinely used in visual research.

\section{THE SYSTEM}

The course is built around a Lab 8/I computer and the PS/8 FOCAL language (Schneider, Smith, \& Whitney, 1971). A block diagram of the system in its current version is shown in Figure 1. The computer is equipped with an $8 \mathrm{~K}$ core memory and operates under OS/8 control, an RK08 disk unit serving as the system device. Two peripherals, an X-Y plotter and a Teletype, are key ones since the teaching methods are interactive and involve graphical analysis of data. In addition, an interface device, called a PT08, connects the computer through telephone lines to the computer center of the University. It may be used by advanced students to perform more elaborate plotting operations than are included in the main body of the course. Besides these, the computer is equipped with several other peripheral devices. These are used for response averaging applications that have been described elsewhere (Armington, 1972).

Connecting the $\mathrm{X}-\mathrm{Y}$ plotter to the computer through the AX08 is a simple task. The AX08 is a flexible interfacing device, equipped with D-to-A converters for driving an oscillograph display. The deflection terminals on the $\mathrm{X}-\mathrm{Y}$ plotter are merely connected across those of the

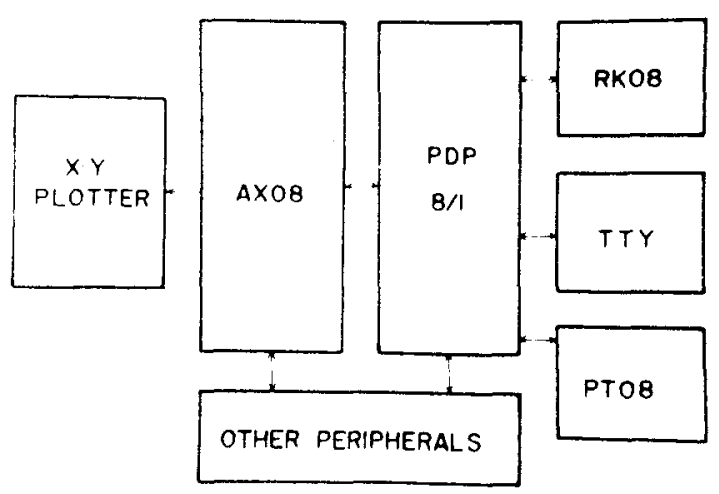

Figure 1. Block diagram of the computer system used for instruction in quantitative methods. 


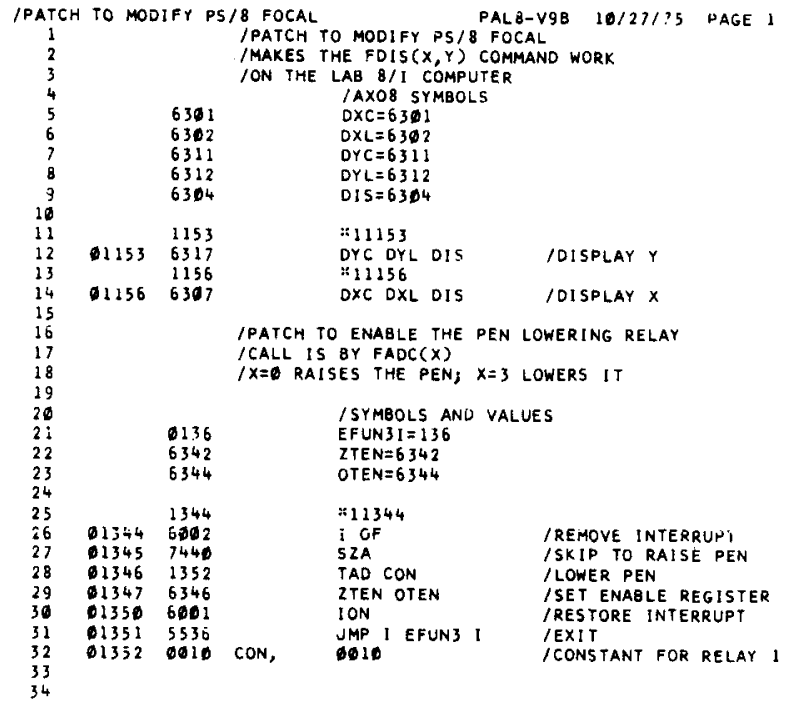

Figure 2. PAL listing of the patches to PS/8 FOCAL to adapt it to the Lab 8/I plotter.

oscilloscope terminals. The AX08 also contains several relay drivers that are activated through the so-called "enable" register. One of these is used to raise and lower the plotter pen.

A patched version of FOCAL, 1968, named PS/8 FOCAL (Schneider, Smith, \& Whitney, 1971), is used in combination with the OS/8 system for programming. Since the standard version of PS/8 FOCAL does not function correctly with plotting equipment shown in Figure 1, two minor modifications had to be made. Their PAL coding is listed in Figure 2. ${ }^{1}$ The first was merely that of changing the digital to analogue coding so that it was compatible with the AX08 rather than the graphics terminal for which $\mathrm{PS} / 8$ was originally prepared. The second was adding a command to raise and lower the X-Y plotter pen. Several alternatives were considered for this, but it was decided to replace the FADC function, originally designed for analogue-to. digital conversion, with new coding. The original does not work with and has no application to the present computer system. As a result of these two changes, the pen is moved to different coordinate values with the command set $\mathrm{H}=$ FDIS $(\mathrm{X}, \mathrm{Y})$ as in standard versions of FOCAL. The command set $\mathrm{H}=\mathrm{FADC}$ (3) lowers the $\mathrm{X} \cdot \mathrm{Y}$ plotter pen and the command set $\mathrm{H}=\mathrm{FADC}(0)$ raises it again.

\section{THE COURSE}

The overall plan of the course is presented in Figure 3. The left column shows the sequence in which various mathematical topics are studied; the right column shows the computer programming that is used to back them up. The course opens with a review of algebra that emphasizes matrices, solution of equations, and functional relations. This is material with which most students have had some previous experience but may have forgotten. To review effectively, it is essential to work problems and solve exercises. This is done with the computer. Students are introduced to FOCAL language at the outset and within a short time are able to write programs for solving a variety of simple problems. Programs are written for computing means, standard deviations, correlations and simultaneous equations, and simple functional relations are plotted. Computer topics match or follow just behind those of the class.

After this introduction, the course proceeds naturally to a consideration of least-squares curve fitting techniques. The classwork is devoted to the derivation and interpretation of the formula which are used for this. Programs are written for actual plotting on the computer. First, straight lines are studied. Then, logarithms, powers, and exponentials are considered, and programs are written to fit fairly complex functions.

The next step is a graphical presentation of calculus. Derivatives of functions are interpreted in terms of their slopes, and the computer is used to plot functions alongside their derivatives. Particular attention is given to exponentials because an understanding of their nature is important for many kinds of research. Finally, a quick review is made of integration. Using the computer to attain approximate solutions seems to facilitate an understanding of this subject.

Obviously, this course, is demanding because of its diversity. Nevertheless, it can be completed in a semester because most topics are not studied in depth. The goal is

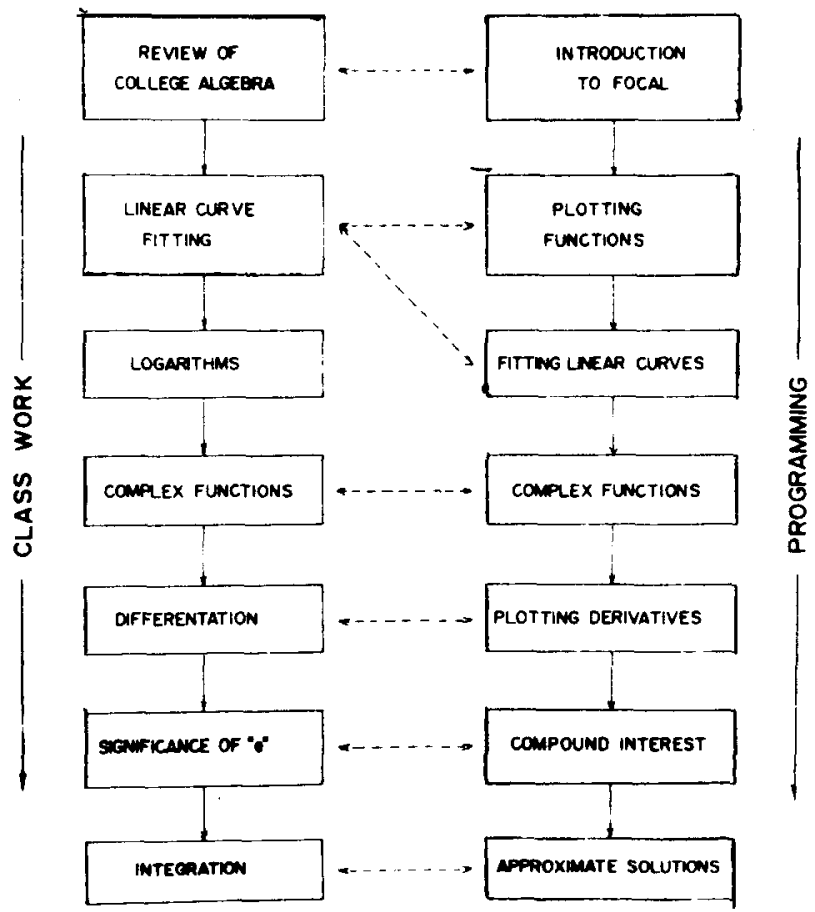

Figure 3. Course outline. This figure shows the sequence of topics. The left column lists the academic subject matter; the right, supporting projects performed on the computer. Dashed arrows point to activities that compliment one another. 


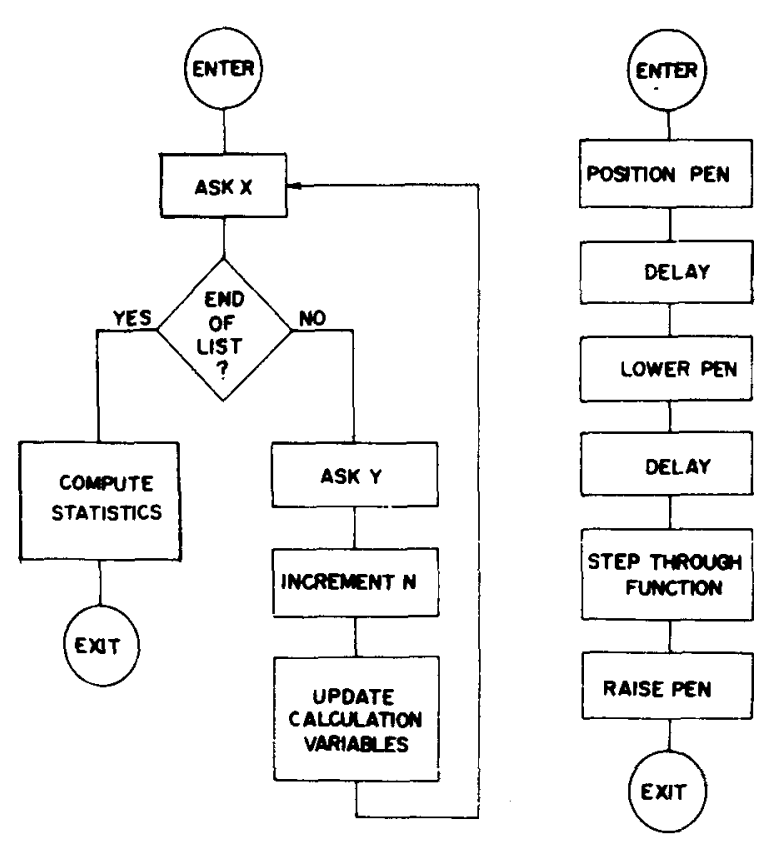

Figure 4. Left: A flow diagram showing the procedure for processing data as it is entered. It prevents the memory area reserved for variables from being overloaded. Right: A flow diagram illustrating the insertion of delay routines to allow the $X-Y$ plotter time to catch up with the signals provided by programs.

to make the meanings of common mathematical operations clear, to equip students to read literature which contains mathematical formulations, and to give them confidence to seek out the analyses most appropriate for their own data. Because the course is conducted with a small number of students (usually 10 to 15 ) and because they work in groups, with the more experienced students helping the less experienced ones, it is possible to achieve a high level of performance in a relatively short time. But, of course, those students who have already had an extensive background in mathematics advance the farthest.

\section{PROGRAMMING TECHNIQUES}

It is a tribute to FOCAL that some of the fairly elaborate computations of the sort that enter into this course can be handled successfully. Several simple techniques make it possible to handle problems that might otherwise require larger computers. A particularly important one is processing data as it is entered, so that a large number of values need not be stored in the memory. An example for entering pairs of values $(X, Y)$, as when a correlation coefficient is being determined or when a curve is being plotted, is shown in the left side of Figure 4. The program asks for successive data points. They are used immediately to update terms such as the sums, sums of squares, and crossproducts that will enter into the final calculation. Then they are discarded. The operator signals the end of the list by typing a word such as "END" after all the data is entered. The IF instruction of FOCAL is used to check each $\mathrm{X}$ entry, and, if END is typed, the program jumps out of the data entry loop. The coding might appear as follows: IF (X-øEND) $A, B, A$, where $A$ is the address of the data processing loop and $\mathrm{B}$ is the address to which the program goes after data entry is complete. It is seldom necessary to retain more than a dozen variables for most common statistical operations regardless of the lengths of the original data lists. Of course, very large quantities of data can still be handled when immediate processing is not feasible by storing them on the disk. Also, because PS/8 makes provision for chaining, it is possible to write extremely long programs by automaticaliy calling up successive sections from the disk as they are needed.

Plotting also requires special consideration. Delays must be inserted into programs to allow the X-Y plotter time to reach points before the pen is lowered. Otherwise, the final result will be smeared. One technique for delay is to use the FOR command: FOR $\mathrm{A}=1$, B; SET $\mathrm{C}=\mathrm{C}+1$, where $\mathrm{B}$ determines the length of the delay and $A$ and $C$ are dummy variables. A continuous line may be drawn by positioning the pen, delaying before lowering it, moving it through the function in small steps, and then raising it. Some of the steps for plotting are shown to the right in Figure 4.

The output of a plotting program must be properly scaled because there is no automatic provision for this in FOCAL. The digital-to-analogue converter of the present system only recognizes $X$ values from 0 to 500 and $Y$ values from -250 to +250 . Thus, when the data does not fit within this range, it must be multiplied by appropriate factors and may need to be offset by constants. Although scaling is easy in concept, students sometimes find it challenging. It provides a good drill with ratios and proportions.

\section{CURVE}

An outcome of the course has been a series of programs that have practical application in the author's laboratory. An example is the generalized program for curve fitting that is called "Curve." The program accepts a set of data, applies calibration factors, plots it, and then fits one or several functions. The fitted curves are drawn through the data points and an index of goodness of fit is obtained. The total program consists of a number of chained subprograms that are stored on the disk and that can be called in flexible sequence, as shown on the left side of Figure 5. When the program is first activated, the experimenter is asked if he needs help, and, if so, the program prints a set of directions for its use. Following this, the experimenter may call up a program which plots a grid having a number of divisions that is set for the particular set of data at hand. Then one of several routines, which calibrate the data in different ways, is used to enter data from the Teletype 


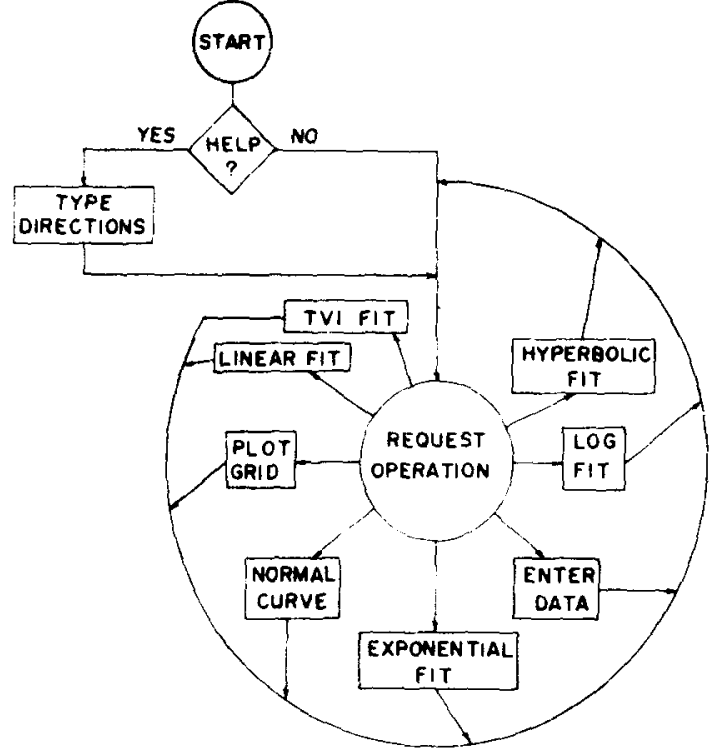

and to store them on the disk. Provision is made for correcting typing errors. Next, any of several subprograms for fitting the data may be called. They plot the points, as well as the fitted curve, and type out a statistical description of the result, including the coefficient of determination which is taken as an index of goodness of fit. Linear, power, logarithmic, exponential, or hyperbolic curves may be fitted singly or in turn to see which gives the best result. Several special routines are used for fitting data from visual experiments. One, for example, fits curves to differential threshold data (TVI data from color adaptation experiments). Another is useful for fitting amplitude-luminance data from electroretinographic experiments. Branching from one to another of these subprograms is achieved by using the ASK instruction to request the name of the desired subprogram, and then using the IF instruction to test the operator's response. This structure is virtually the same as that used to test for the end of a list of data, as described above.

Other practical programs solve simultaneous

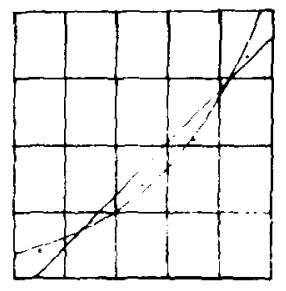

Figure 5. Left: A flow diagram for a generalized curve fitting program. Each block within the circular portion is a separate subprogram that is stored on the disk. They may be called in any sequence to find the function that seems to fit the data best. Right: Two plots produced by this program. In the upper plot, a straight line and an exponential have been fitted to a set of experimental points. The exponential gives a better fit. The lower right-hand section shows a histogram constructed by placing test scores in 10 class intervals and the normal curve which best fits them. equations, rank order data, plot histograms, and plot best-fitting normal curves. Two examples of the output of the Curve program are shown at the right side of Figure 5.

\section{REFERENCES}

Armington, J. C. Using the Lab-8 for experimentation with the human visual system. Behavior Research Methods \& Instrumentation. 1972. 4, 61-63.

DANIELS, F. Mathematical preparation for physical chemistry. New York: McGraw-Hill, 1928. New copyright 1956.

GuIf ford. J. P. Psychometric methods. New York: McGrawHill. 1936. New copyright 1954.

Lewts. D. Quantitative methods in psychology. Iowa City: University of lowa. 1966.

Schneider, D.. Smith, B., \& Whitney, R. PS $/ 8$ FOCAL, 1971. Portland. Oregon: Oregon Museum of Science and Industry, 1971.

Smith. C. A. B. Biomathematics (Vols. I, II). Neu York: Hafner. 1966.

\section{NOTE}

1. The coding for these patches was developed by Steve Reef. 